\title{
3-D Free Surface Simulation in the Electromagnetic Dam
}

\author{
Katsuaki MORITA, Mitsuhiro YOSHIDA, Chie KUWADA, Shinya KANAMORI, Hiroshi NAKAJIMA and \\ Takahiko TANAHASHI ${ }^{11}$
}

Hiroshima Research \& Development Center, Mitsubishi Heavy Industries, Ltd., Kan-on-shin-machi, Nishi-ku, Hiroshima 7338553 Japan. 1) Department of Mechanical Engineering, Keio University, Hiyoshi, Kohoku-ku, Yokohama $223-8522$ Japan.

(Received on October 30, 2002; accepted in final form on January 28, 2003)

\begin{abstract}
A method to study the physical phenomena of the molten metal under the AC magnetic field has been required to design the electromagnetic (EM) dam system. This confines a side of the molten metal by Lorentz force without contact between the side wall and the molten metal. In this work, we have developed a numerical analysis method to predict the 3-D phenomena of the molten metal considering the free surface. To evaluate steady the state of the molten metal including the free surface, the integrated computational errors are eliminated after certain iteration step. This process avoids the solution diverged and provides the converged steady state solution. However, although the error elimination process neglects the physical phenomena of smaller scale than the elimination time step related, this method could predict slowly varying the surface shape obtained by the experiment. By using this method, the effects of magnetic field direction and the EM boundary condition on the surface shape are investigated. The profile of Lorentz force corresponding to the direction of magnetic field and boundary condition was found to affect the flow pattern resulting in the change of the steady state surface shape.
\end{abstract}

KEY WORDS: electromagnetic field; Lorentz force; eddy current; free surface; electromagnetic dam; molten metal; edge element; volume of fluid; simulation.

\section{Introduction}

Use of electromagnetic (EM) dam plays a major role in the evolution of the steel industry. ${ }^{1,2)}$ The molten metal is pushed away from a side wall by Lorentz force under the AC field, so that both the life extension of the side wall and the purification of products with less contamination could be achieved.

The AC magnetic field applied parallel to the surface of molten metal inside the conductive pot, induces the eddy current interacting with the original magnetic field, which generates Lorentz force to push the surface away from the side wall. ${ }^{3)}$ The surface shape is changed by Lorentz force, accordingly the EM field profile changes near the surface. Thus these phenomena interacting the EM field and the flow field with the free surface are quite complex. In the equilibrium state, the EM pressure, the hydrostatic pressure, and the hydrodynamic pressure are balanced at the surface.

It is required to form the free surface with lower power loss in order to make this technique possible. Because the edge of the conductive pot is also exposed by the EM field, the eddy current loss at the conductive pot could not be ignored. ${ }^{1)}$ To design a commercial scale EM dam system, the computational technique, which predicts and explains physical phenomena, has been necessary. The EM and the fluid field considering the free surface shape should be coupled in a calculation. However, it has been taken enormous computing time to calculate the complete and precise interac- tive phenomena (especially, the iteration of 3-D EM field calculation). An alternative way called shadow method was proposed $^{4)}$ to reduce the computing time, which calculates Lorentz force considering the free surface transition based on the analytical approximation. In the shadow method, new Lorentz force is evaluated as a function of clearance between the coil and the molten metal with one numerical calculation of the EM field at the first stage. This method is suitable for a simple system consisted of the coil and the molten metal without the core or the conductive pot. When the core or the conductive pot is set in a system, it is difficult to evaluate accurate 3 -D Lorentz force distribution by the shadow method. In such a system, the numerical calculation is required to consider the 3-D boundary conditions with time varying free surface.

A 2-D model has been developed ${ }^{5)}$ in which the field calculations have been coupled completely with the free surface shape and the dynamic effect of the molten metal flow in a coreless induction furnace. Although the 2-D model is suitable for calculating within shorter computing time, the simple extension of this model to the 3-D system needs enormous computing time to obtain steady state.

Another author ${ }^{6)}$ approximately calculated the surface shape of the molten metal in the cold crucible system. EMfield is estimated as the surface of the molten metal changes. However, the balance of the steady EM pressure and the hydrostatic pressure evaluated the surface shape of the molten metal without considering dynamical behavior caused by the flow of the molten metal. The EM field was 
calculated by the 3-D finite elemental method (FEM) with edge elements. The method with edge elements ${ }^{7)}$ takes significantly less computing time than the traditional method with nodal elements, which could be utilized in a new model. Cha and Hwang ${ }^{8)}$ predicted the surface shape of the EM slab caster with the similar 3-D method using the iteration of EM calculation. However, the effect of hydrodynamic pressure was not taken into account to evaluate the surface shape.

Numerical computational method for the free surface of the fluid flow is classified into two groups. The first is to use self-adjusting grids. For example, Evans and Kageyama $^{9}$ simulated the free surface of the EM casting by ALE (Arbitrary Lagrangian-Eulerian) method. This method would not be suitable, if the surface shape changes significantly or folds over itself. The second is the static grid method called VOF (Volume Of Fluid). In this method, the significant surface change could be handled, but smearing of the surface due to the numerical diffusion is occasionally a problem. Kohno et al. ${ }^{10)}$ applied this method to calculate the simple 3-D free surface system with the EM field. This level set method, which reinitialize the free surface shape every certain interval, could be used to prevent smearing of the surface.

Most of these numerical computational methods have calculated the coreless induction furnace without the conductive pot. Some numerical results with the core and the conductive pot have published, ${ }^{11)}$ but the iteration between the EM and the fluid field has not been included. Therefore, the main aim of this work is to develop a numerical calculation method for the 3-D EM dam system with the complete EM and fluid coupling applying the level set method to VOF and to clarify the physical phenomena of the molten metal.

\section{System Layout}

Figure 1 shows an EM-dam system. The AC magnetic field, which is generated by the AC coil current with the high permeability core, is applied parallel to the molten metal surface (The coil is not shown in Fig. 1). The magnetic field induces the eddy current on the surface of molten metal, which interacts with the original field. This interaction induces Lorentz force, which pushes the surface away. There are two options to confine the molten metal inside the conductive pot, one is the horizontal magnetic field and the other is the vertical magnetic field (Figs. 1(a) and 1(b)). Both cases have been tested to investigate the effect of magnetic field direction. Figure 1(c) shows the size of the conductive pot in this work. One side of the pot has open boundary. The triangular cross section type pot shown in Fig. 1(d) was tested to investigate the EM sealing property for more realistic system of the EM dam ${ }^{1)}$ at which magnetic field crosses over the inclined boundary.

\section{Computer Modeling}

\subsection{Assumptions}

The modeling of EM dam was based on the following assumptions:

(1) Fluids were Newtonian and incompressible;

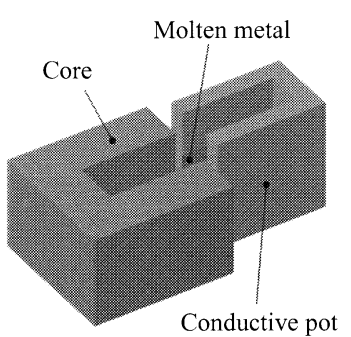

(a)

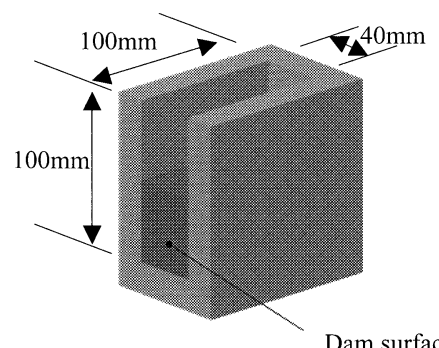

(c)

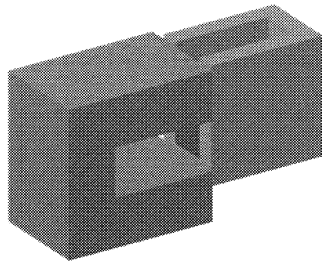

(b)

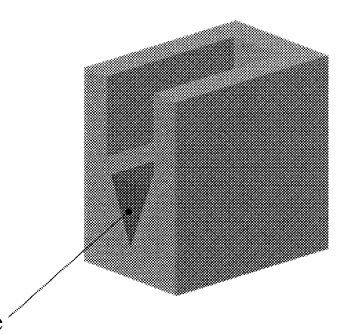

(d)
Fig. 1. EM-dam systems including core and conductive pot: (a) horizontal field system; (b) vertical field system; (c) rectangular pot; (d) triangular pot.

(2) The material characteristics were constant;

(3) The electric field induced by the interaction of fluid velocity and the magnetic field was negligible;

(4) The pressure due to the surface tension could be negligible from the result of order-estimation;

(5) The temperature distribution in the molten metal was uniform.

\subsection{Numerical Modeling}

The EM field was calculated by the 3-D FEM with edge elements using the $A-\phi$ method. The fundamental equations are given as follows:

$$
\begin{aligned}
& \nabla \times\left(\frac{1}{\mu} \nabla \times A\right)=J_{\mathrm{o}}-\sigma\left(\frac{\partial A}{\partial t}+\nabla \phi\right) \\
& \nabla \cdot \sigma\left(\frac{\partial A}{\partial t}+\nabla \phi\right)=0
\end{aligned}
$$

where $\mu$ is the magnetic permeability, $A$ is the magnetic vector potential, $J_{\circ}$ is the source current density, $\sigma$ is the conductivity and $\phi$ is the electric scalar potential.

Since $A$ varies sinusoidally in the EM-dam system, the time derivative of $A$ is replaced by the complex function $j \omega A$, where $\omega=2 \pi f$ ( $f$ : frequency). In addition, the matrix of the FEM is solved by the ICCG (Incomplete Cholesky Conjugate Gradient) method.

Lorentz force density $F$, which is generated by the interaction of the induced currents and the applied magnetic field, is expressed as:

$$
F=\sigma\left(\frac{\partial A}{\partial t}+\nabla \phi\right) \times(\nabla \times A)
$$

The fluid field calculation was based on Navier-Stokes equation and the equation of continuity for both the air and the molten metal: 


$$
\begin{array}{r}
\rho \frac{\partial u}{\partial t}+\rho u \cdot \nabla u=-\nabla p+\eta \nabla^{2} u+F+\rho g \\
\nabla \cdot u=0 \ldots \ldots \ldots \ldots \ldots \ldots \ldots \ldots \ldots \ldots \ldots \ldots \ldots \ldots
\end{array}
$$

where $\rho$ is the mass density, $u$ is the velocity, $p$ is the pressure, $\eta$ is the viscosity and $g$ is the gravitational acceleration.

The 3-D transient finite-volume solution algorithm is selected with well known $k-\varepsilon$ model for the turbulence modeling.

The scalar value called VOF (Volume of Fluid) was used for the free surface modeling. The mass density of each calculative cell for two fluid layers (air and molten metal) is:

$$
\rho=\rho_{1}(1-C)+\rho_{2} C
$$

where $\rho_{1}$ is the mass density of air, and $\rho_{2}$ is the mass density of molten metal. $C$ is a scalar value called VOF, which expresses the volume ratio, defined as follows:

$$
\begin{gathered}
C=\frac{V_{2}}{V_{1}+V_{2}} \\
0 \leq C \leq 1
\end{gathered}
$$

where $V_{1}$ is the volume of air and $V_{2}$ is the volume of molten metal.

The transport equation is described as follows under the assumption of constant density of both fluids:

$$
\frac{\partial C}{\partial t}+\nabla C u=0
$$

\subsection{Coupling Methods}

The flow chart of this model is shown in Fig. 2, which is developed to integrate the 3-D calculations of the EM field, the fluid field and the free surface shape of the molten

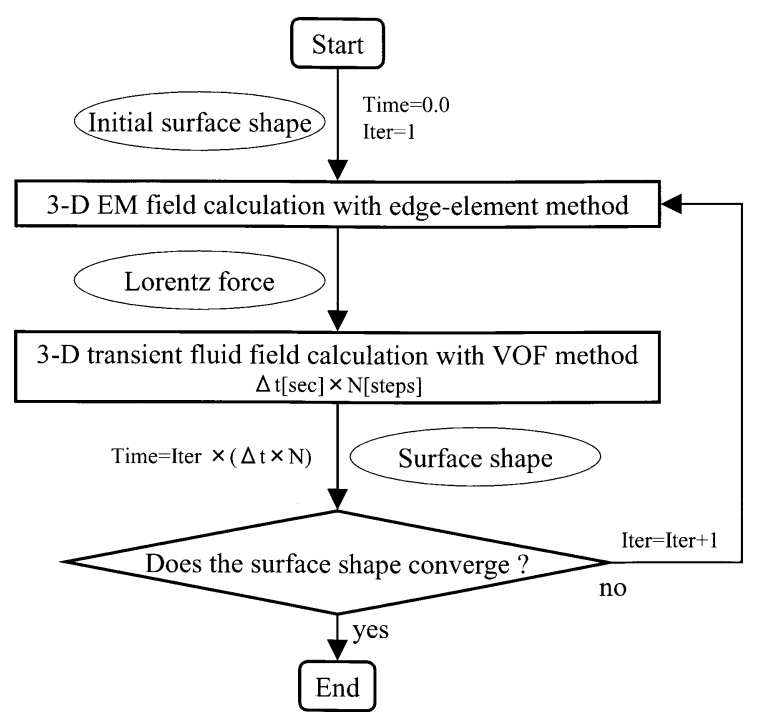

Fig. 2. The flow chart of the coupling method between EM and fluid field calculation: where Time is the transient time for simulated scale; Iter is an integer to count the number of iterations of the EM calculation; $\Delta t$ is the time step size for fluid calculation; and $N$ is a constant integer which is the number of steps of fluid calculation per one EM calculation. metal. The UNIX shell script was created to run two commercial software packages (MAGNA from CRC Solutions Corp., Japan, for the EM field calculation, and STAR-CD from CD-adapco Japan Corp., for the fluid flow calculation).

The EM field calculations are repeated every $N$-step transient fluid calculation, which results in the reduction of computing time. $N$ is determined as such, the surface shape slightly changes during $N$-step fluid calculation. Lorentz force $F$ is evaluated from the last EM calculation result $F_{\mathrm{o}}$ by using the value of VOF, which enables as to estimate the force acting on the surface:

$$
F=C F_{\mathrm{o}} \text {. }
$$

To evaluate the surface shape for the next EM calculation after $\mathrm{N}$-step fluid calculation, the value of VOF is reinitialized in each cell as:

$$
\begin{array}{lll}
C=1.0 & \text { (if } & 0.5 \leq C) \\
C=0.0 & \text { (if } & C<0.5)
\end{array} .
$$

This re-initialization is similar to the level set method, ${ }^{10}$ ) however this method is much simpler, just setting new $C$ values. The re-initialization eliminates integrated computational errors, which prevents diverging or smearing the surface of molten metal. However, it should be noted that reinitialization neglects the physical phenomena, which are smaller scale than the elimination time step related. Therefore, the validity of this re-initialization process should be confirmed by the experimental data.

Figure 3 shows typical grids of EM dam with the vertical magnetic field. Half of the region is calculated under the symmetrical condition. The EM region is divided properly with tetrahedral elements including the air region. The cells that would include the free surface are divided into smaller grids. These smaller grids could deal with the skin depth effect. The fluid region could be simply divided with hexagonal elements. To share the data of Lorentz force and the free surface position between the EM and fluid calculation, the data-mapping method is used, in which each grid of fluid calculation takes the value of Lorentz force from a grid of EM calculation that covers the center of the grid of fluid calculation.

\subsection{Computational Setup}

The calculations were carried out using Silicon Graphics server, Origin 3000. Conditions of the applied field and the physical properties of materials are listed in Table 1. The parameters of EM dam are listed in Table 2.

\section{Experimental Conditions}

The experiment was carried out to verify the computational results. The apparatus of the EM dam is shown in Fig. 4. Either horizontal (Fig. 4(a)) or vertical (Fig. 4(b)) field was applied by rotating magnet. The U-Alloy 70 from Osaka Asahi Metal Ltd, Japan was used for the molten metal because of its low melting temperature, $70^{\circ} \mathrm{C}$. The components of the U-Alloy 70 are $\mathrm{Bi}(50 \%), \mathrm{Pb}(27 \%), \mathrm{Sn}$, $\mathrm{Cd}$, and In. The temperature of copper pot was controlled to be constant around $80^{\circ} \mathrm{C}$ by circulating hot water. Insulator for the dam-side wall was necessary to prevent the leakage 


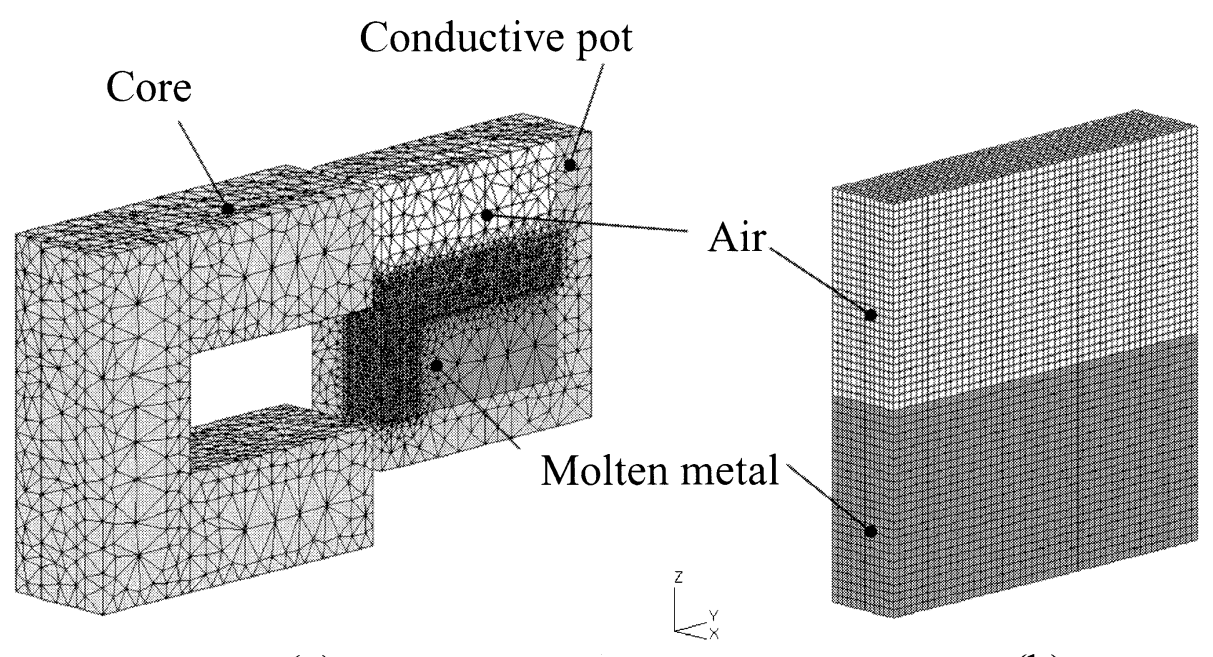

(a)

(b)

Fig. 3. Typical grids of EM dam with vertical magnetic field for the rectangular pot: (a) grid for EM calculation; (b) grid for fluid calculation.

Table 1. Conditions of computational setup.

\begin{tabular}{|c|c|c|c|}
\hline \multirow{2}{*}{ Coil } & Operating current & (A-turns) & 4500 \\
\hline & Frequency & $(\mathrm{kHz})$ & 10 \\
\hline \multirow{3}{*}{ Core } & Material & & Silicon steel sheets \\
\hline & Electric conductivity & $(\mathrm{S} / \mathrm{m})$ & 0.0 \\
\hline & Relative permeability & $(\cdot)$ & 150 \\
\hline \multirow{3}{*}{ Pot } & Material & & Copper \\
\hline & Electric conductivity & $(\mathrm{S} / \mathrm{m})$ & $5.00 \times 10^{7}$ \\
\hline & Relative permeability & $(-)$ & 1.0 \\
\hline \multirow{5}{*}{ Air } & Material & & Air \\
\hline & Electric conductivity & $(\mathrm{S} / \mathrm{m})$ & 0.0 \\
\hline & Relative permeability & $(\cdot)$ & 1.0 \\
\hline & Mass density & $\left(\mathrm{kg} / \mathrm{m}^{3}\right)$ & 1.205 \\
\hline & Viscosity & $(\mathrm{kg} / \mathrm{ms})$ & $1.81 \times 10^{-5}$ \\
\hline \multirow{5}{*}{ Molten metal } & Material & & U-Alloy 70 \\
\hline & Electric conductivity & $(\mathrm{S} / \mathrm{m})$ & $1.06 \times 10^{6}$ \\
\hline & Relative permeability & $(\cdot)$ & 1.0 \\
\hline & Mass density & $\left(\mathrm{kg} / \mathrm{m}^{3}\right)$ & 9700 \\
\hline & Viscosity & $(\mathrm{kg} / \mathrm{ms})$ & $1.86 \times 10^{-3}$ \\
\hline
\end{tabular}

Table 2. Parameters of EM dam.

\begin{tabular}{llll}
\hline & EM-field direction & Pot type & Initial pool height $(\mathrm{mm})$ \\
\hline Case1 & Vertical & Rectangular & 50 \\
Case2 & Horizontal & Rectangular & 50 \\
Case3 & Vertical & Triangular & 40 \\
\hline
\end{tabular}

of molten metal when the EM field was off. Silicon steel sheets were used for the core material that was temperaturecontrolled by water-cooled copper plates.

The shape of the surface was measured by two methods. The thermo sensible paper, which changes color at certain temperature, was used, inserting into the molten metal. The surface profile was clearly obtained as the pattern on this paper. This first method could be used for the horizontal EM field (vertical eddy-current field). For the vertical EM field, the insulation paper insulates the horizontal eddy current. Another method is to use a foil made of the same material as the molten metal. The foil inserted melts leaving outside of the molten metal and the surface shape was ob-

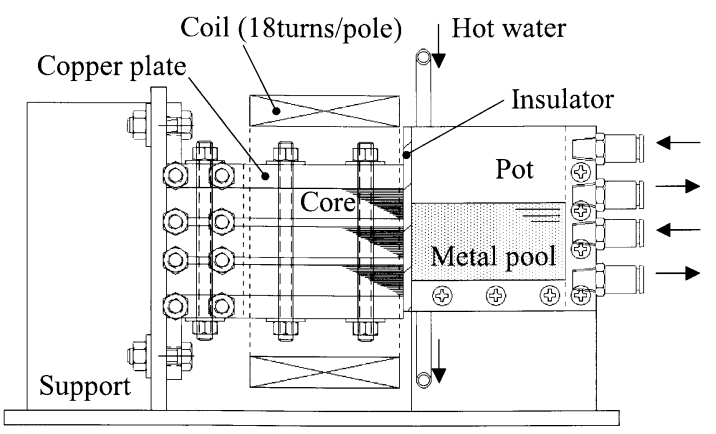

(a)

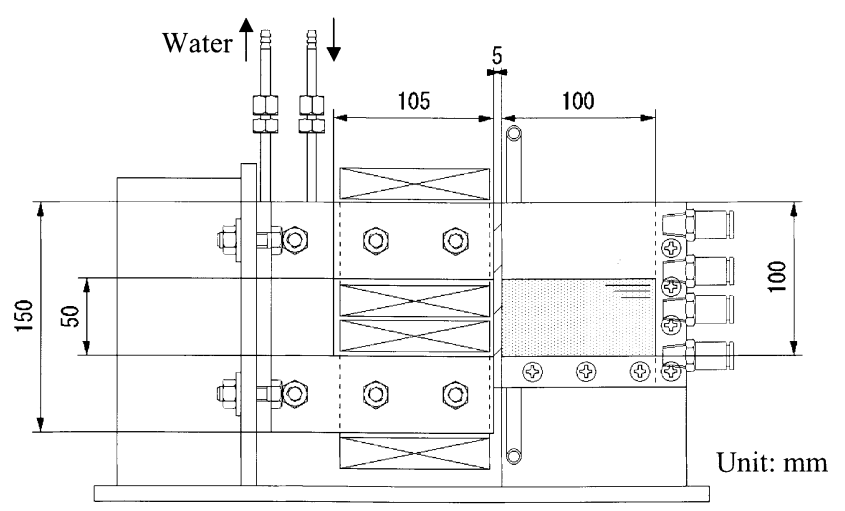

(b)

Fig. 4. Side view of the experimental apparatus of EM dam: (a) horizontal setup; (b) vertical setup.

tained.

In all cases, the EM dam was conducted at $10 \mathrm{kHz}$ and 4500 ampere-turns shown in Table 1 . The experimental parameters were set as shown in Table 2.

\section{Results and Discussion}

\subsection{Computing Time}

100 iterations of EM calculations were repeated until it is converged. The EM field calculation has been repeated every 20-step of fluid calculations, where the time step $\Delta t$ 
Table 3. Number of grids and computing time.

\begin{tabular}{llllllllc}
\hline & \multicolumn{3}{c}{ Number of grids for EM calculations } & \multicolumn{3}{c}{ For fluid calculations } & \multicolumn{3}{c}{ CPU time (hours) } \\
& Nodes & Cells & Edges & Nodes & Cells & EM & + & Fluid \\
\hline Case1 & 18819 & 105594 & 126713 & 40824 & 36517 & 80 & + & 5 \\
Case2 & 18854 & 106508 & 127431 & 40824 & 36517 & 80 & + & 5 \\
Case3 & 16324 & 90694 & 109284 & 46805 & 42930 & 60 & + & 7 \\
\hline
\end{tabular}

Computer used: SGI Origin 3000

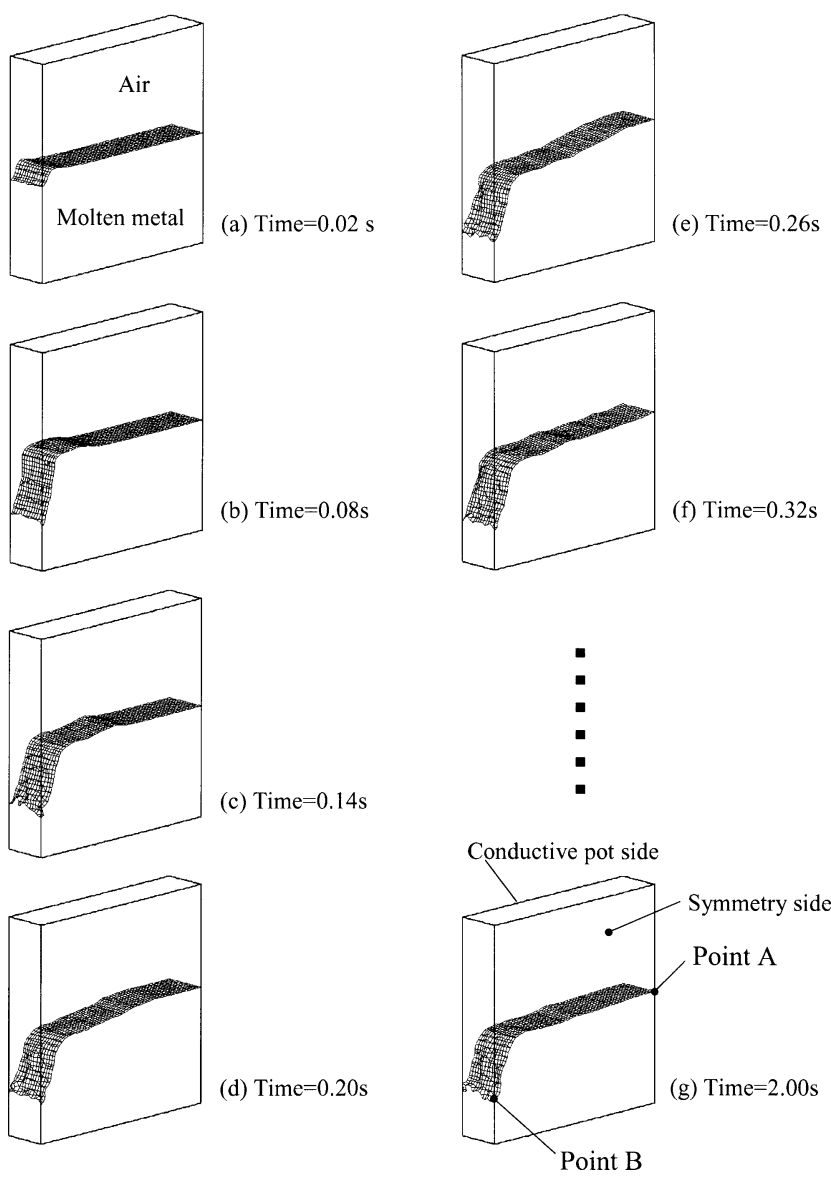

Fig. 5. Typical calculated result of time evolution of 3-D surface shape in Case 1.

was fixed $0.001 \mathrm{~s}$. The number of grids and total computing time for 100 iterations are listed in Table 3. One calculation step of the EM field takes about 48 (Case 1 and Case 2) or 36 (Case 3) minutes, resulting 80 or $60 \mathrm{~h}$ for total computing time for 100 iterations. By use of the edge-element method for the EM calculation, the computing time becomes shorter comparing with many iterations of other 3-D calculation. The computing time for the fluid calculation is much shorter for each step.

\subsection{Time Evolution of Surface Shape}

Figure 5 shows typical calculation results of the time variation of 3-D fluid including the surface shape for Case 1. A wave induced by the initial Lorentz force is simulated traveling on the surface of molten metal. The steady state shape is obtained in $2 \mathrm{~s}$ when the wave becomes weaker. The time variation of the molten metal surface height at Point A and Point B, which are indicated in Fig. 5(g), is shown in Fig. 6. Point B is defined as the contact point on the symmetry plane. From the figure, it is confirmed that the system is converged and reached to be steady up to $2 \mathrm{~s}$.

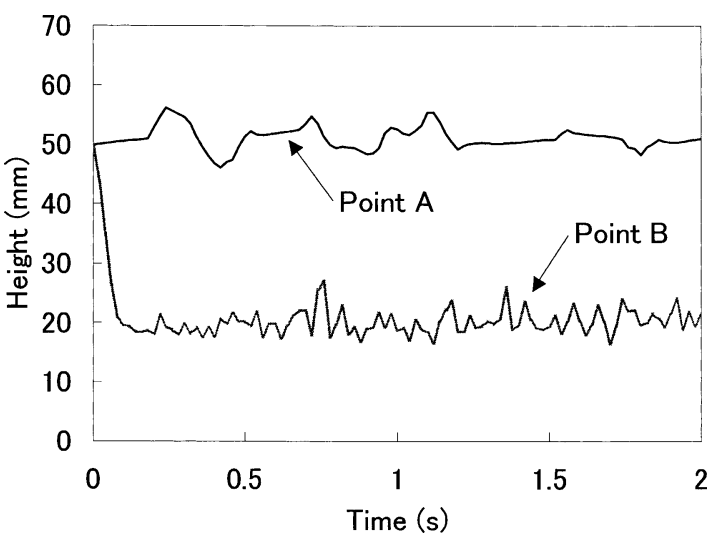

Fig. 6. Time variation of molten metal height in Case 1 at Point A and Point B that are indicated in Fig. 5(g).

Table 4. Qualitative comparison of the physical phenomena in Case 1 and Case 2.

\begin{tabular}{lll}
\hline & Case 1 & Case2 \\
\hline Direction of Magnetic field & Vertical & Horizontal \\
Direction of Eddy current & Horizontal & Vertical \\
\hline Eddy current in conductive pot & Small & Large \\
Eddy current in molten metal & Large & Small \\
Lorentz force in molten metal & Large & Small \\
No-contact height & High & Low \\
Power loss in conductive pot & Small & Large \\
\hline Vertical gradient of Lorentz force & Large at middle & Small at top \\
Flow pattern & Two loops & One loop \\
\hline
\end{tabular}

\subsection{Effects of Magnetic Field Direction}

The effects of magnetic field direction and the EM boundary condition are investigated in detail. The simulated EM and fluid field distributions in Case 1 and Case 2 are compared with each other. The qualitative comparison is summarized in Table 4.

\subsubsection{No-contact Height}

Figures 7(a) and 7(b) show calculated and measured surface shapes on the symmetry plane with different magnetic field direction for Case 1 and Case 2. Calculated results satisfactorily agree with the measured results. Some discrepancies were occurred in Case 2, which was considered some errors due to the instability at Point B or measurement error. No-contact height is defined as the difference between the height of Point A and Point B. As for the comparison between Case 1 and Case 2, it is found that the nocontact height in Case 1 is higher than that in Case 2 in spite of the same coil current condition. This means that vertical magnetic field is more desirable to achieve higher no-contact height. 


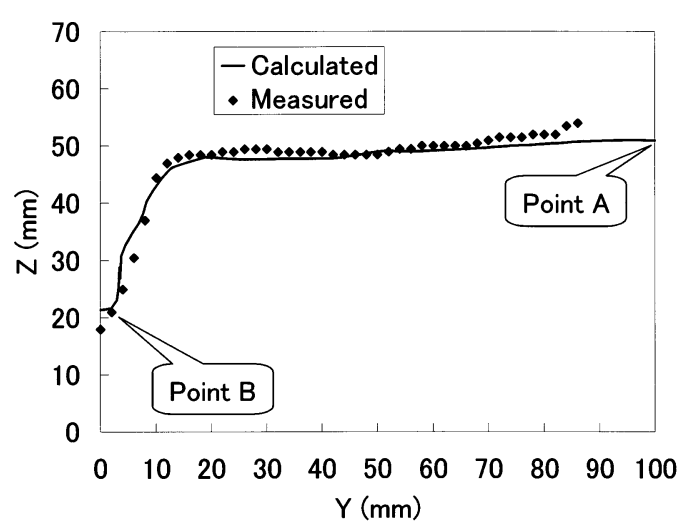

(a) Case1.

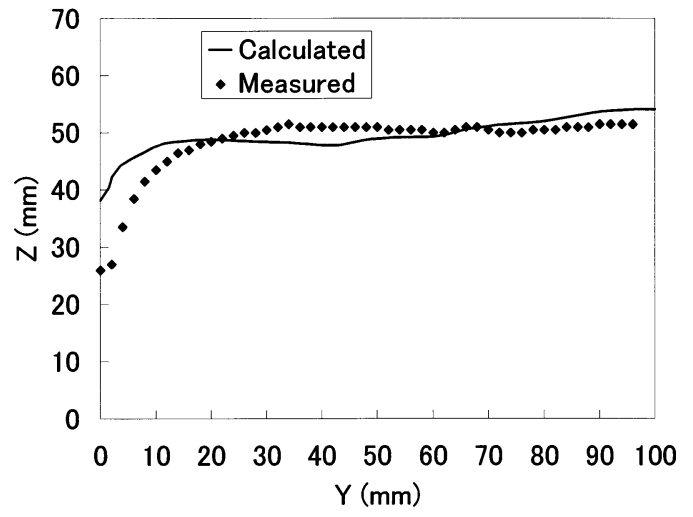

(b) Case2.

Fig. 7. Quantitative comparison between calculated and measured steady state surface shapes in Case 1 and Case 2 .
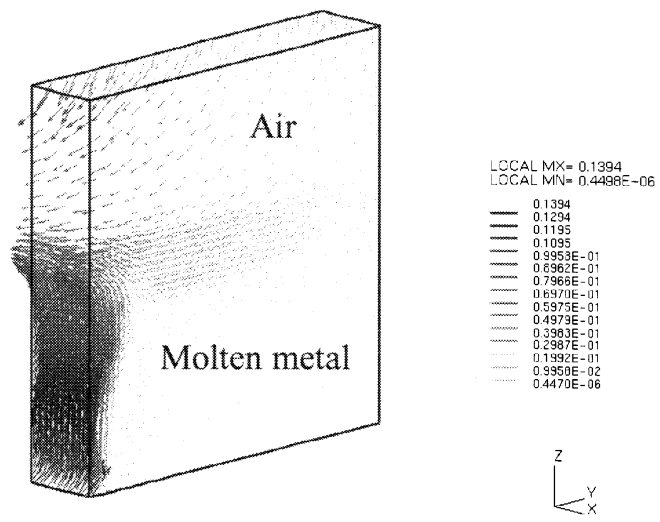

Magnetic flux density, Max. $0.14 \mathrm{~T}$

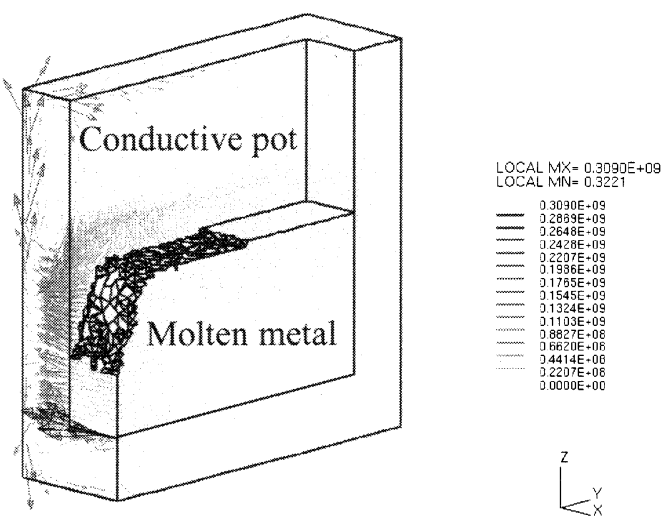

Eddy current density, Max. $3.1 \times 10^{8} \mathrm{~A} / \mathrm{m}^{2}$

(a) Case1.

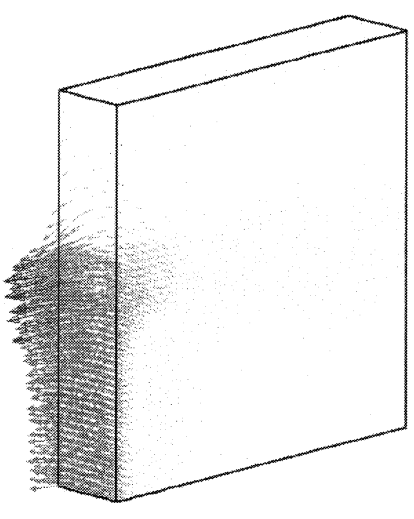

Magnetic flux density, Max. $0.24 \mathrm{~T}$
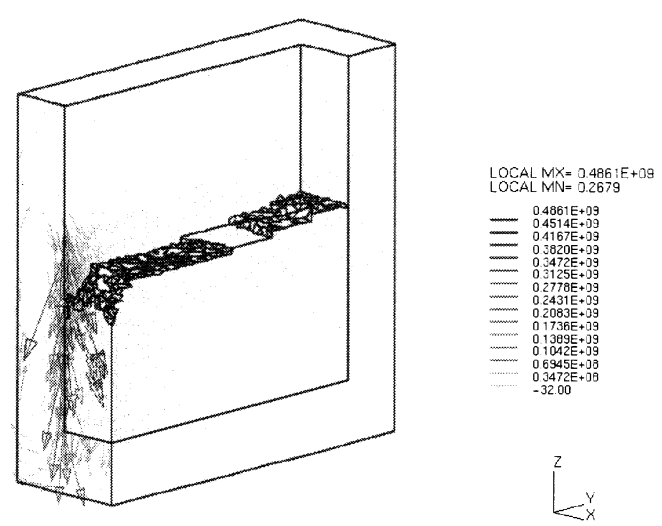

Eddy current density, Max. $4.9 \times 10^{8} \mathrm{~A} / \mathrm{m}^{2}$

(b) Case2.

Fig. 8. Steady state distributions of magnetic flux density and eddy current density in Case 1 and Case 2 .

\subsubsection{Magnetic Flux and Eddy Current}

The left side of Fig. 8 shows the distribution of the magnetic flux density in the molten metal and upper air region in Case 1 and Case 2. The magnetic flux concentrates along the molten metal surface by the skin effect. The right side of Fig. 8 shows the distribution of the eddy current density in the conductive pot. The eddy current flows perpendicular to the magnetic flux. Therefore the direction of eddy cur- rent at the edge of conductive pot is horizontal in Case 1 and vertical in Case 2. The maximum value in Case 1 $\left(3.1 \times 10^{8} \mathrm{~A} / \mathrm{m}^{2}\right)$ is much smaller than that in Case 2 $\left(4.9 \times 10^{8} \mathrm{~A} / \mathrm{m}^{2}\right)$. In Case 2 , the eddy current is easier to flow, because the direction of the edge of the conductive pot boundary is the vertical direction, which is same as eddy current direction. On the other hand, in Case 1, there is a horizontal discontinuous boundary condition of eddy cur- 


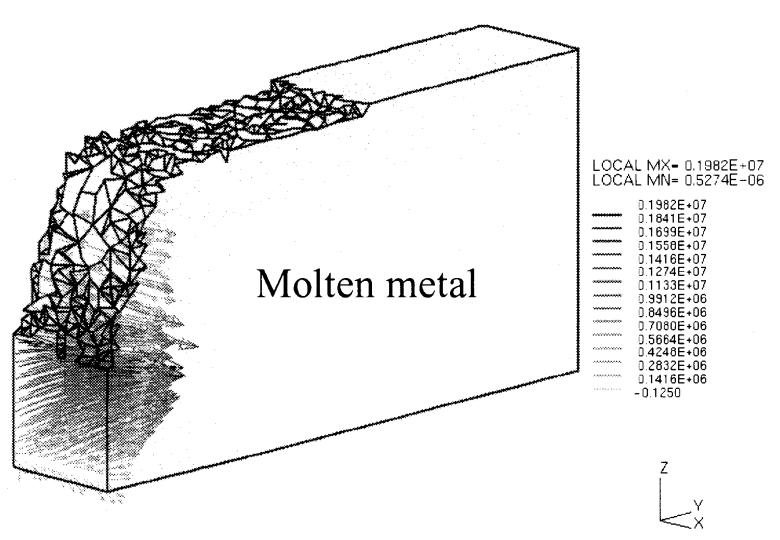

Lorentz force density, Max. $2.0 \times 10^{6} \mathrm{~N} / \mathrm{m}^{3}$

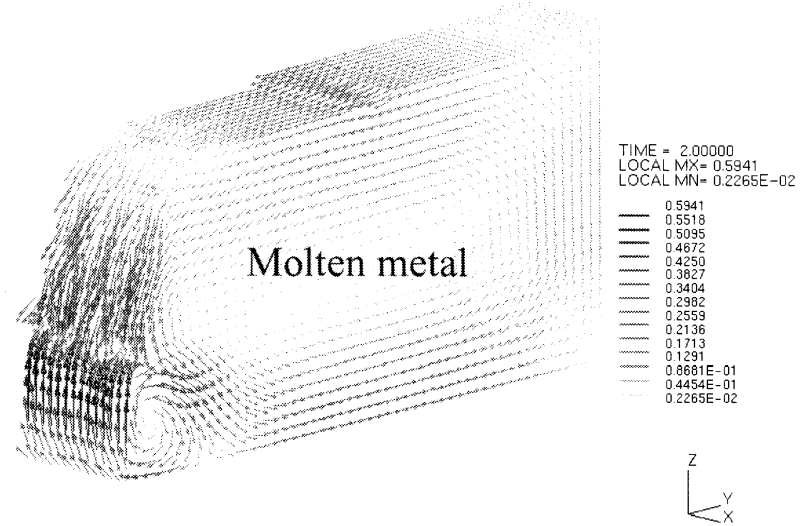

Flow velocity, Max. $0.59 \mathrm{~m} / \mathrm{s}$

(a) Case1.

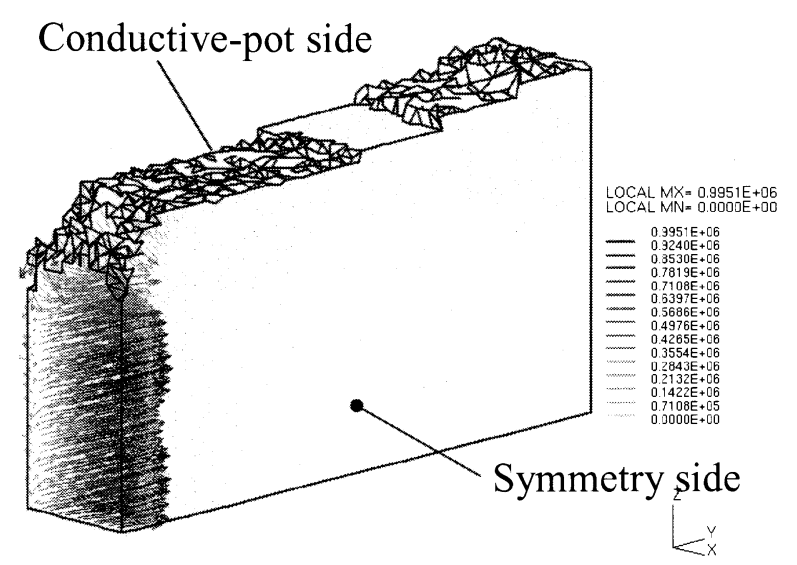

Lorentz force density, Max. $1.0 \times 10^{6} \mathrm{~N} / \mathrm{m}^{3}$

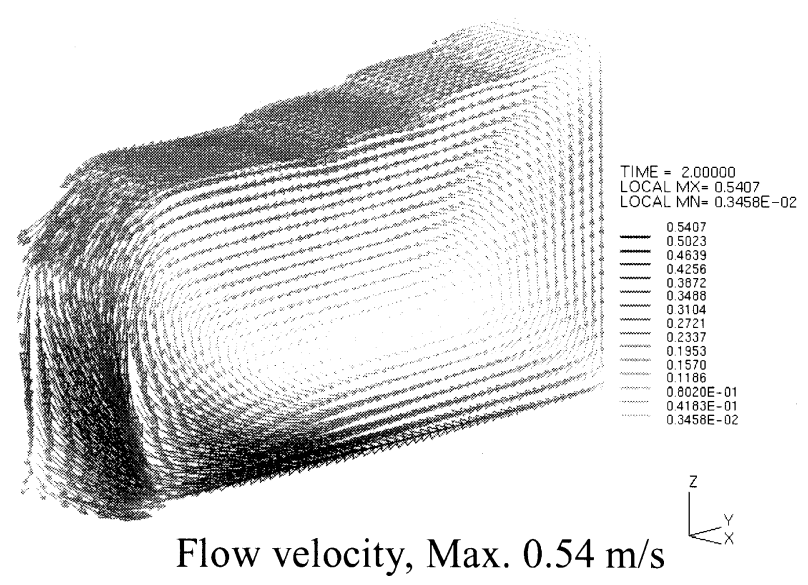

(b) Case2.

Fig. 9. Steady state distributions of Lorentz force density and flow velocity in Case 1 and Case 2 .

rent at the interface of the conductive pot edge when the molten metal is pushed away. This means that Lorentz force profile generated by $\mathrm{AC}$ magnetic field is affected by the electric boundary condition through eddy current profile.

\subsubsection{Lorentz Force and Flow Pattern}

The left side of Fig. 9 shows the distribution of Lorentz force density in the molten metal in Case 1 and Case 2. The maximum value is $2.0 \times 10^{6} \mathrm{~N} / \mathrm{m}^{3}$ in Case 1 , and $1.0 \times 10^{6}$ $\mathrm{N} / \mathrm{m}^{3}$ in Case 2. The force in Case 2 is smaller than that in Case 1 , because the eddy current is mainly focused on conductive pot in Case 2 shown in Fig. 8, reducing the eddy current in the molten metal. This calculation agrees with the result of higher no-contact height in Case 1 than that in Case 2. The power loss at the conductive pot in Case 1 is lower than that in Case 2 because the eddy current in Case 1 is smaller than that in Case 2. Thus, the vertical magnetic field is superior to the horizontal field on high no-contact height with lower power loss.

The vertical gradient of Lorentz force greatly affects the flow pattern of the molten metal. In Case 1, the eddy current in the molten metal has horizontal profile, which is relatively reduced at the bottom of the metal pool. This reason is considered as such, the part in the conductive pot near the bottom of the metal pool is the easiest part to flow for the eddy current. Lorentz force is also reduced at the upper part of the surface, because of the distance between the magnet and the molten metal surface, which is pushed away. The result of Case 1 shows that Lorentz force is large in the middle height of the pool. As for in Case 2, the vertical gradient of Lorentz force is almost uniform except the upper part where the molten metal is pushed away. Fluid flow velocity distributions in Case 1 and Case 2 are shown on the right side of Fig. 9. The flow directions along the molten metal surface are directed from a small part of Lorentz force to a large part. In Case 1, two different rotating loops are formed. This might be caused by the vertical gradient of Lorentz force that is large in the middle height. The confluent point of two loops is near the contact point between the molten metal and the wall of dam side. This point might be limiting the no-contact height. As for the flow pattern in Case 2, one-loop flow from the top to the bottom on the surface is formed due to the vertical gradient of Lorentz force. The flow from the conductive pot side to the symmetry side is also formed due to the horizontal gradient of Lorentz force. These results indicate that the profile of Lorentz force is important for flow pattern, which might be affecting to the surface stability. 


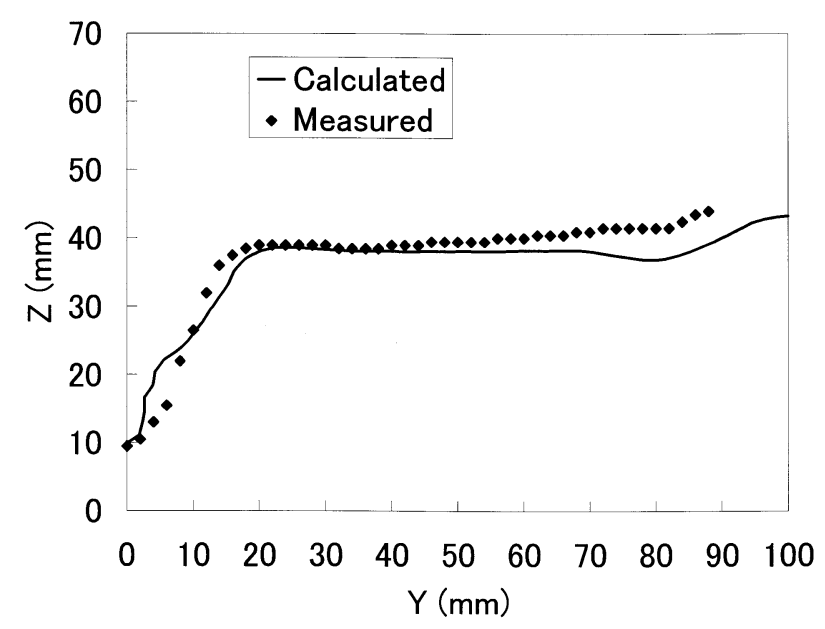

(a)

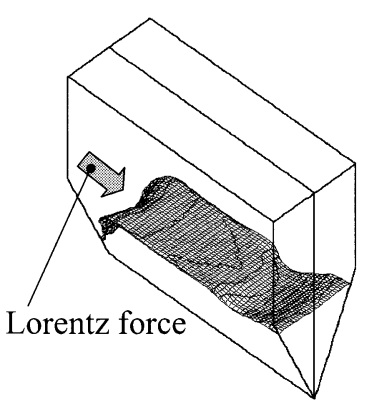

(b)

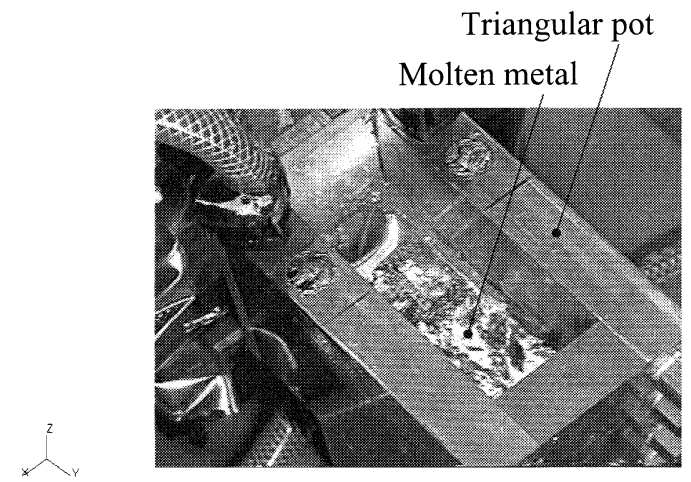

(c)

Fig. 10. Calculated and experimental results in Case 3: (a) comparison between the calculated and measured steady state surface shape on the symmetry plane; (b) top view of the calculated 3-D steady state surface shape; (c) top view of the observed 3-D surface shape in the experiment.

\subsection{Triangular Cross Section}

The triangular cross section pot type with the vertical magnetic field was also tested in Case 3. The results are shown in Fig. 10. Figure 10(a) compares the calculated and measured surface shape on the symmetry plane in Case 3. The calculated shape matches quite well with the experimental measurement. No-contact height in Case 3 is obtained as high as in Case 1. The 3-D shape in Case 3 was compared qualitatively in Figs. 10(b) and 10(c). The 3-D calculated shape from the top view has good agreement with the experimental camera view. The calculation method in this work is also verified for the general system of EM dam with the inclined pot boundary.

\section{Conclusion}

A numerical analysis method to predict the 3-D phenomena of the molten metal considering the free surface in the EM dam has been developed. 3-D surface shapes were simulated and the steady state surface shape was obtained by eliminating the integrated computational errors after certain iteration step. The calculated surface shapes matched well with the experimental results. By using this method, it has been made possible to study the change of surface shape according to control parameters. The direction of the applied magnetic field greatly affects to the eddy current profile at the conductive boundary. The vertical magnetic field was found superior to the horizontal field on resulting in high no-contact height and less power loss. The gradient of Lorentz force affects the flow pattern of the molten metal, which might be limiting no-contact height. The vertical magnetic field is also effective to keep high and stable nocontact height for different cross section of molten metal.

\section{REFERENCES}

1) K. E. Blazek, W. F. Praeg. J. G. Rachford, Y. H. Wang and M. Mohri: Iron Steelmaker, 25 (1998), 39.

2) H. Gerber, K. Blazek, Y. Wang and W. Praeg: Proc. of the 3rd Int. Symp. on Electromagnetic Processing of Materials, 2000, ISIJ, Tokyo, (2000), 241.

3) M. Yoshida, K. Morita, C. Kuwada and T. Tanahashi: Proc. of Int. Seminar on Heating by Internal Sources (HIS-01), ed. by S. Lupi et al., Univ. of Padua, Padua, (2001), 425.

4) K. Fujisaki and T. Ueyama: J. Appl. Phys., 83 (1998), 6356.

5) X. R. Zhu, R. A. Harding and J. Campbell: ISIJ Int., 39 (1999), 1.

6) Y. Kawase and T. Yoshida: IEEE Trans. Magnetics, 35 (1999), 1889.

7) A. Kameari: Applied Electromagnetics in Materials, Proc. of the 1st Int. Symp., Tokyo, (1988), 225.

8) P. R. Cha, Y. S. Hwang, H. S. Nam, S. H. Chung and J. K. Yoon: ISIJ Int., 38 (1998), 403.

9) J. W. Evans and R. Kageyama: Proc. of the 3rd Int. Symp. on Electromagnetic Processing of Materials, 2000, ISIJ, Tokyo, (2000), 295.

10) H. Kohno, T. Tanahashi, K. Morita and M. Yoshida: Proc. of the 13th Symp. on Electromagnetics and Dynamics (The 13th SEAD), JSME, Tokyo, (2001), 354.

11) F. C. Chang, L. R. Turner, J. R. Hull, Y. H. Wang and K. E. Blazek: Proc. of Steelmaking Conf., ISS, Warrendale, PA, (1998), 589. 